\title{
Regularities of process of disaggregating material particles in dynamic separator with disaggregating device
}

\author{
Vladimir Trofimchenko \\ Belgorod State Technological University named after \\ V.G. Shukhov \\ BSTU named after V.G. Shukhov \\ Belgorod, Russia \\ 308012, Kostukova St., 46 \\ trofimchenko@inbox.ru
}

\author{
Sergey Khanin \\ Belgorod State Technological University named after \\ V.G. Shukhov \\ BSTU named after V.G. Shukhov \\ Belgorod, Russia \\ 308012, Kostukova St., 46 \\ sergiykhanin@gmail.com
}

\begin{abstract}
To improve the efficiency of the separation of powdered materials which are prone to aggregation, internal separator devices are used that contribute to the disaggregate material particles. For the most effective destruction aggregates of particle during physical contact with devices, it is necessary to establish rational values of their parameters. For the disaggregating device in the form of multi-pass belt, an expression was developed that makes it possible to calculate the rational angle of the installation of the belt, on the basis of the vector components of the velocity of the aggregate just before contact with the belt. An analytical expression is obtained for determining the efficiency of a disaggregation process by the multi-pass belt disaggregating device in a dynamic separator, and a regression equation that allows one to determine the rational values of design parameters of the device. Practical results were obtained in determining the efficiency of the particle disaggregation process, which are comparable with the values of analytical calculations.
\end{abstract}

Keywords - dynamic separator, disaggregating device, powder materials, aggregates of particles, efficiency of disaggregation

\section{INTRODUCTION}

The building materials industry is an industry that ensures the development and growth of the country's economy. In the production of building materials, the production of powdered materials by grinding is an energy-intensive process. Grinding complexes with a separator are used for the production of powders with the specified granulometric composition and specific surface area. A significant problem in the production of powdered materials is aggregation (physical contact of the particles under the influence of forces of different origin), which is typical for materials which are prone to adhesion. To a large extent, aggregation is inherent in fine particles, while the aggregate size of such an aggregate can often correspond to the size of the coarse fraction. As a consequence, the fine product particles in the aggregate are repeatedly returned to the mill as coarse particles. The presence of fine particles (finished product) in a coarse fraction of particles leads to a decreasing of the grinding complex efficiency and causes energy losses. At the moment, selectivity (the probability of entering of particles to a coarse product) of the best separators reaches only $15 \%$ for particles less than $5 \mu \mathrm{m}$ [1]. Consequently confirmed the relevance of the particles aggregation problem for powder materials production. At the same time, the study of particles aggregation and creating of devices that improve efficiency of disaggregation process are actual tasks.

Significant contribution to the study of powdered material separation processes, their disaggregation and the development of calculation techniques have been made: [2-8] as well as others. Their work and research have contributed significantly to the study of the separation process, the problems associated with the aggregation of powder materials and approaches to disaggregation. The problems of mechanical destruction of bonds between particles in aggregates and the patterns of change in efficiency of the disaggregation process depend on parameters of the disaggregating device, rational values of the parameters themselves did not receive sufficient study.

\section{METhODOLOGY}

In separators, the process of interaction of aggregates of particles with devices under certain conditions leads to the disaggregation of particles. The efficiency of this process depends on the design parameters of the devices and dynamic characteristics of the machine at the moment of contact. The multi-pass belts disaggregating device contribute to the effective destruction of aggregates due to the optimal angle of installation of the belts. The values of the components (radial, tangential, vertical) of the particle velocity equivalent to the constituent velocities of the aggregate $\left(\mathrm{w}_{\mathrm{r}}-\right.$ radial, $\mathrm{w}_{\varphi}-$ tangential, $\mathrm{w}_{\mathrm{z}}$ - vertical) allow one to determine the energy of interaction with the disaggregating device, and also to establish the optimum angle of installation for multi-pass belts for destruction the aggregates of various sizes. 
Immediately before contact with the surface of the multipass belts, which are installed in the separation chamber, the aggregate equivalent to the particle size has the following kinetic energy $T$, J:

$$
\mathrm{T}=\frac{\mathrm{m}}{2}\left(\mathrm{w}_{\mathrm{r}}^{2}+\mathrm{w}_{\varphi}^{2}+\mathrm{w}_{\mathrm{z}}^{2}\right) .
$$

where $\mathrm{m}$ is the mass of the aggregate particle, $\mathrm{kg}$.

In [9] eq. (2) is used to find the energy of disaggregation during grinding:

$$
\frac{\eta_{\mathrm{u}} E}{\mathrm{~m}}=\frac{\mathrm{i} \sigma}{3 \rho}\left(\mathrm{S}_{\kappa}-\mathrm{S}_{\mu}\right) .
$$

where $\sigma$ is surface tension at the boundary "grindable particles - surrounding liquid medium», $\mathrm{J} / \mathrm{m}^{2}$;

$\mathrm{S}_{\kappa}, \mathrm{S}_{H}$ is specific surface area of the particles respectively before and after grinding, $\mathrm{m}^{2} / \mathrm{m}^{3}$; $\rho$ is particle density, $\mathrm{kg} / \mathrm{m}^{3}$

$\eta_{\text {и }}$ is efficiency of a grinding machine, in fractions of a unit;

$E$ is the energy imparted to the material to be grinded from outside, $\mathrm{J}$;

$\mathrm{m}$ - mass of material to be grinded, $\mathrm{kg}$;

$\mathrm{i}$ is the degree of grinding, which is determined by equation:

$$
\mathrm{i}=\frac{\mathrm{S}_{\kappa}}{\mathrm{S}_{H}}
$$

Taking into account eq. (2), it is possible to obtain an equation for determining the efficiency of the disaggregation process by the disaggregating device in dynamic separator. We assume that the specific surface areas before disaggregation and after disaggregation during separation are determined by the following relations:

$$
\mathrm{S}_{b d}=\frac{\pi d_{a}^{2}}{\frac{\pi d_{a}^{3}}{6}}=\frac{6}{d_{a}}
$$

where $d_{a}$ is initial aggregate diameter, $\mathrm{m}$;

$$
\mathrm{S}_{a d}=\frac{\pi \bar{d}_{k}^{2}}{\frac{\pi \bar{d}_{k}^{3}}{6}}=\frac{6}{\bar{d}_{k}} ;
$$

where $\bar{d}_{k}$ is average final particle diameter after disaggregation, $\mathrm{m}$.

Eq. (4) and (5) allow us to determine the degree of disaggregation $\mathrm{k}$ during separation.

$$
k=\frac{\mathrm{S}_{a d}}{\mathrm{~S}_{b d}}=\frac{d_{a}}{\bar{d}_{k}}
$$

If to use eq. (1) to describe the energy transferring to the aggregate from the outside during separation, then, taking into account (4) and (5), we obtain following equation:

$$
\frac{4 k \sigma_{a}}{\rho}\left(\frac{1}{\bar{d}_{k}}-\frac{1}{d_{a}}\right)=\eta\left(\mathrm{w}_{\mathrm{r}}^{2}+\mathrm{w}_{\varphi}^{2}+\mathrm{w}_{\mathrm{z}}^{2}\right),
$$

where $\eta$ is the efficiency of the disaggregation process by multi-pass belts;

$\sigma_{a}$ is surface tension at the boundary of an «aggregate gaseous atmosphere», $\mathrm{J} / \mathrm{m} 2$.

In such a manner, taking into account eq. (4), (5) and (7), is possible to count the efficiency of the disaggregation process for the device during separation in percent:

$$
\eta=\frac{4 \sigma_{a}\left(d_{a}-\bar{d}_{k}\right)}{\rho \bar{d}_{k}^{2}\left(\mathrm{w}_{\mathrm{r}}^{2}+\mathrm{w}_{\varphi}^{2}+\mathrm{w}_{\mathrm{z}}^{2}\right)} 100 \%
$$

Eq. (7) taking into account (6) takes the following form:

$$
\eta=\frac{4 \sigma_{a} k(k-1)}{\rho d_{a}\left(\mathrm{w}_{\mathrm{r}}^{2}+\mathrm{w}_{\varphi}^{2}+\mathrm{w}_{\mathrm{z}}^{2}\right)} 100 \% .
$$

Eq. (8) and (9) show that the efficiency of the destruction of two identical aggregates depends on the speed of the aggregate and will be maximum when installing disaggregating multipass belts installed at an angle perpendicular to the velocity vector of the aggregate (Fig. 1).

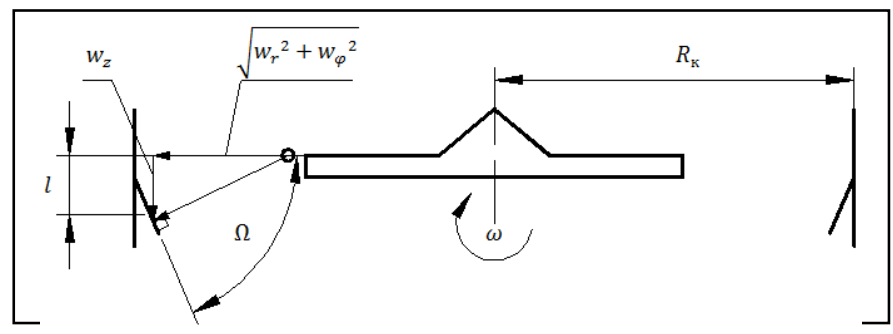

Fig. 1. Scheme for calculating the installation angle of disaggregating multipass belt

According to the calculation scheme, the value of the angle of installation of the belt to ensure a frontal impact on the surface of a multi-pass belt for different values of the diameters of the material particles relative to the horizon will be determined by the following relationship [10]:

$$
\Omega=\frac{\pi}{2}-\operatorname{arctg} \frac{\mathrm{W}_{\mathrm{z}}}{\sqrt{\mathrm{w}_{\mathrm{r}}^{2}+\mathrm{w}_{\varphi}^{2}}} .
$$

Eq (10) makes it possible to calculate the installation angles of disaggregating multi-pass belts relative to the horizontal plane, depending on the value of the components (vectors) of the particle velocity $\mathrm{W}_{\mathrm{r}}, \mathrm{W}_{\varphi}, \mathrm{W}_{\mathrm{z}}$. Assuming that the velocity of the particles corresponds to the velocity of equivalent aggregates size, according to eq. (10), it is possible 
to determine the rational angle of installation belts of the disaggregating device.

\section{RESULTS}

The components of particle velocity are key parameters for determining the interaction energy of aggregates with a disaggregating device and for determining the design parameters of such disaggregating devices in the form of multiple-pass belts. Table 1 presents the values of the velocity components of particle aggregates of different sizes.

TABLE I. Components of the velocity of aggregates for the separator «Polydor» $\varnothing 4000$

\begin{tabular}{|c|c|c|c|c|}
\hline Size, $\mu \mathrm{m}$ & $\mathbf{8 0}$ & $\mathbf{2 0 0}$ & $\mathbf{3 1 5}$ & $\mathbf{6 3 0}$ \\
\hline $\mathrm{W}_{\mathrm{r}}, \mathrm{m} / \mathrm{s}$ & 0.41 & 0.94 & 0.95 & 0.97 \\
\hline $\mathrm{W}_{\varphi}, \mathrm{m} / \mathrm{s}$ & 0.11 & 0.88 & 1.3 & 1.85 \\
\hline$\left|\mathrm{W}_{\mathrm{z}}\right|, \mathrm{m} / \mathrm{s}$ & 2.1 & 0.5 & 0.63 & 0.82 \\
\hline
\end{tabular}

Using the values of the velocity vectors $\mathrm{w}_{\mathrm{r}}, \mathrm{w}_{\varphi}, \mathrm{w}_{\mathrm{z}}$, it is possible to calculate the angle of the velocity vector when the particle of the material comes to a fragment of the multipass belt after its departure from the surface of the distributor plate and, taking this into account, the optimum installation angle of multi-pass belt, in which the destruction of the aggregates will be maximum (the front contact of the aggregates with a belt).

Table 2 shows the results of the calculation of the angle of installation of multi-pass belts in the separator "Polidor" $ø 4000$ at a constant air flow rate of $30 \mathrm{~m}^{3} / \mathrm{s}$.

TABLE II. Angle of installation of multi-pass belts for different size particles

\begin{tabular}{|l|c|c|c|c|}
\hline Size, $\boldsymbol{\mu m}$ & $\mathbf{8 0}$ & $\mathbf{2 0 0}$ & $\mathbf{3 1 5}$ & $\mathbf{6 3 0}$ \\
\hline$\Omega$, degree & 11 & 84 & -75 & -66 \\
\hline
\end{tabular}

It is determined that the angle of installation of the belts relative to the horizontal surface of the distributor plate for particles of size $315 \mu \mathrm{m}$ is in the absolute value of 75 degrees and 66 degrees for $630 \mu \mathrm{m}$.

Fig. 2 shows the dependence of the efficiency of the disaggregation $\eta$ on the degree of disaggregation $k$.

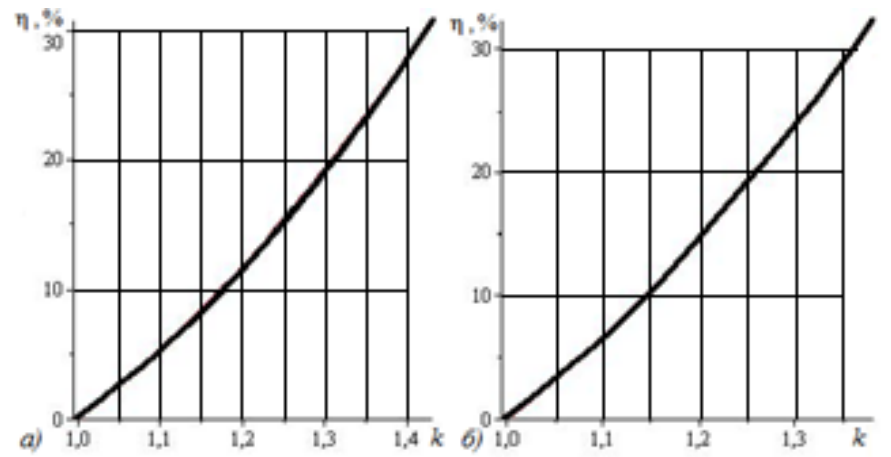

Fig. 2. Dependence of the efficiency of the process of disaggregation on the degree of disaggregation for initial aggregates of size a) $80 \mu \mathrm{m}$ and b) $200 \mu \mathrm{m}$
The diagrams are constructed in accordance with the design and technological parameters of the dynamic separator "Polydor" ø4000, with air flow through the separation chamber of $30 \mathrm{~m} / 3$. The value of the efficiency disaggregation $\eta=29 \%$ for the aggregate of $80 \mu \mathrm{m}$ achieves at a degree of disaggregation $k=1.42$, for an aggregate of size $200 \mu \mathrm{m}$, the same value is reached for $k=1.35$. These values show that interacting energies are different and depend on masses of aggregates of different sizes.

Figure 3 shows the dependence of the efficiency of the disaggregation process on the degree of disaggregation of the initial aggregate. The graphs are constructed for a dynamic separator "Polydor" ø4000, with airflow through the separation chamber of $30 \mathrm{~m}^{3} / \mathrm{s}$. The value of the efficiency of the process of disaggregation $\eta=30 \%$ for an aggregate of 315 $\mu \mathrm{m}$ corresponds to the value of the degree of disaggregation $k=1.72$; for an aggregate of $630 \mu \mathrm{m}$, the efficiency value of $30 \%$ corresponds to the degree of disaggregation $k=2.48$.

The obtained dependences show that in order to achieve the same efficiency of the process of disaggregation of different initial aggregates of different sizes, it is necessary to achieve various degrees of disaggregationю The larger the aggregate, the higher the degree of disaggregation.
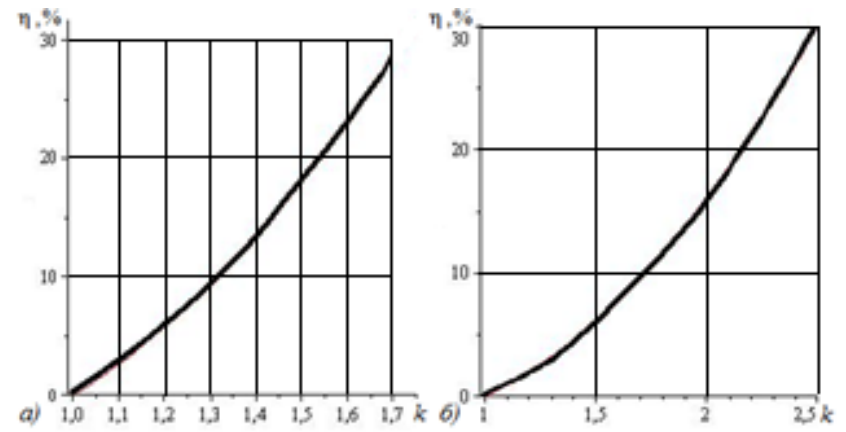

Fig. 3. Dependence of the efficiency of the process of disaggregation on the degree of disaggregation for initial aggregates of size, $\mu \mathrm{m}$ a) 315 and b) 630

Thus, the obtained eq. (8) and (9) allow determining the efficiency of the process of particle disaggregation in a dynamic separator with multi-pass belts of disaggregating device. The graphical dependencies characterize the efficiency of the disaggregation process for different values of the degree of disaggregation.

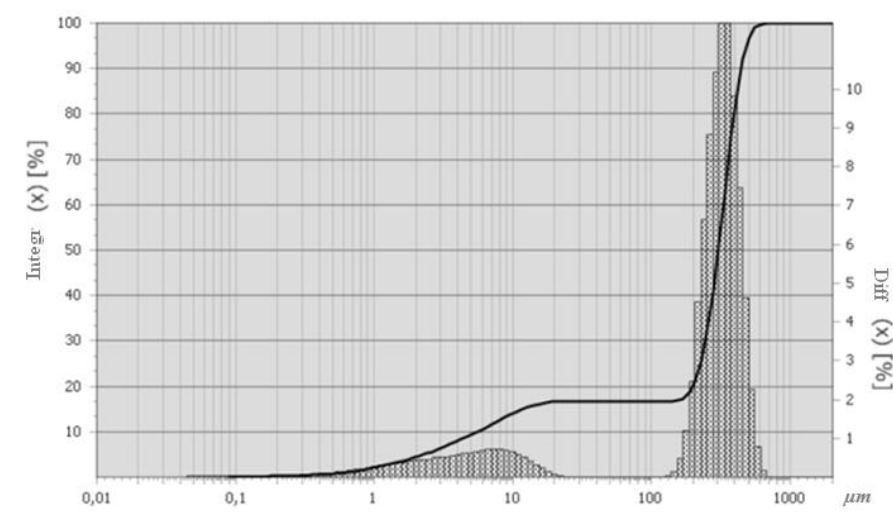

Fig. 4. Particle-size distribution of aggregates for fraction $+200 \ldots-315 \mu \mathrm{m}$ 
To confirm the theoretical results, an experiment was performed at the experimental separator with a disaggregating device to determine the dependence of the disaggregation efficiency on the device parameters. As a material, ground marl was used. Particle-size distribution of aggregates for fraction $+200 \ldots-315 \mu \mathrm{m}$ is shown in Fig. 4.

Graphic dependencies in fig. 5-7, constructed on the basis of the equation (11), made it possible to establish the regularities of the change in the efficiency of disaggregation from the factors studied and to determine the range of their rational values.

The regression equation for determining the efficiency of disaggregation:

$$
\begin{aligned}
& \eta=-172,04+103,84 \cdot \mathrm{H} / \mathrm{h}+7,09 \cdot \mathrm{T} / \mathrm{h}+ \\
& +1068,54 \cdot \mathrm{B} / \mathrm{D}+4,82 \cdot \Omega-0,03 \cdot \Omega^{2}- \\
& -841,77 \cdot(\mathrm{H} / \mathrm{h})^{2}-1,05 \cdot(\mathrm{T} / \mathrm{h})^{2}- \\
& -14295,00 \cdot(\mathrm{B} / \mathrm{D})^{2}+0,30 \cdot \Omega \cdot \mathrm{H} / \mathrm{h}- \\
& -0,01 \cdot \Omega \cdot \mathrm{T} / \mathrm{h}+0,75 \cdot \Omega \cdot \mathrm{B} / \mathrm{D}+5,00 \cdot \mathrm{H} / \mathrm{h} \cdot \mathrm{T} / \mathrm{h}- \\
& -833,33 \cdot \mathrm{H} / \mathrm{h} \cdot \mathrm{B} / \mathrm{D}+3,750 \cdot \mathrm{T} / \mathrm{h} \cdot \mathrm{B} / \mathrm{D}
\end{aligned}
$$

where $H / h$ is the ratio of the installation height of the belts of the device $H$ to the height of the separation chamber $h$;

$T / h$ is the ratio of the screw pitch of the multi-pass belts $T$ to the height of the separation chamber $h$;

$B / D$ is ratio of the width of the bands $B$ to the diameter $D$ of the separation chamber.

Graphic dependencies, constructed on the basis of regression equations, reflecting the patterns of change the $\eta$ from the values of design parameters are expressed in a complex form $(T / h ; H / h ; B / D)$ and $\Omega$.

In Fig. 5 a) there is a change of the disaggregation efficiency in the range from $21.4 \%$ to $29.4 \%$.
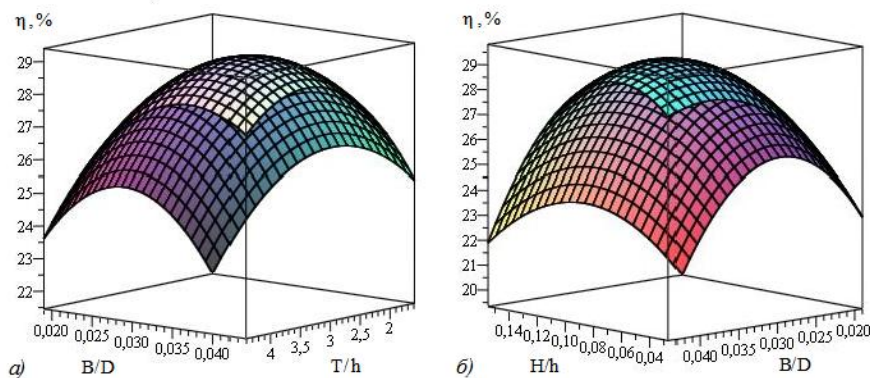

Fig. 5. Dependencies of the efficiency of disaggregation process on parameters: a) $B / D$ and $T / h$, b) $H / h$ and $B / D$

The surface of the function is parabolic and reaches its maximum values close to the central values of the variation factors; for $B / D=0.032$, and for $T / h$ the value at the center is 3 . The growth of the function is observed when the factor values change in the direction of the central values for $B / D$ and $T / h$. The minimum value of the effectiveness of the process of disaggregation comes at the minimum values of the factors. The increase of the disaggregation efficiency occurs with an increase in the value of $T / h$ to 3 due to the fact that with an increase in the pitch of the belts $T$, a step suitable for disaggregation is provided, providing a frontal or near-frontal collision of the unit with belts. An increase of the value of $T / h$ over 3 leads to a decrease in the efficiency of the disaggregation process, since the angle of rise of the disaggregating bands varies from the most effective values for the formation of fine particles due to the destruction of aggregates. As the factor $B / D$ increases to 0.032 , the efficiency of the disaggregation process increases, that is, the width of the belt $B$ increases, and the active contact area with the aggregates increases. But with a further increase in the value of the factor, the efficiency of the disaggregation process begins to decrease smoothly - this indicates a decrease in the number of fine particles formed as a result of contact aggregates with belts. This is because a further increase in the width of the belt $B$ leads to an increase in the residence time of the particles on the surface of the belt during rolling, while collisions of aggregates and their destruction upon contact with the belts are not effective as a result of damping.

In Fig. $5 \mathrm{~b}$ ) the efficiency of the disaggregation process varies from 19.3 to $29.8 \%$. The maximum value of the process of disaggregation reaches at the values of factors $H / h=0.090$ and $B / D=0.033$, with a further increase in the parameter $B / D$ and a decrease in $H / h$, the value of the function decreases. The minimum value is reached with a minimum value of $B / D=$ 0.019 and a maximum value of $H / h=0.155$. The increase in the efficiency of the disaggregation process with increasing $H / h$ is due to the fact that a change in the height $\mathrm{H}$ of the position of the disaggregating device in the separation chamber from 0.027 to 0.100 moves the device to a zone of more intensive interaction with aggregates where the formation of fines particles increases as a result of disaggregation. For factor values $H / h$ above 0.100 , the response function begins to decrease sharply, since the disaggregating device moves above the zone of the most active interaction with the aggregates. Decrease in the efficiency of the disaggregation process also takes place at values of the factor $B / D$ below 0.032 , as this reduces the width of the bands $B$, and hence the contact surface of the device's belts with the units. An increase in $B / D$ above 0.032 leads to a decrease in the efficiency of disaggregation due to damping of the aggregates.

Graphical dependences of the disaggregation efficiency on the combinations of factors $\mathrm{B} / \mathrm{D}$ and $\Omega, \mathrm{T} / \mathrm{h}$ and $\mathrm{H} / \mathrm{h}$ are shown in Fig. 6 a), b), respectively.
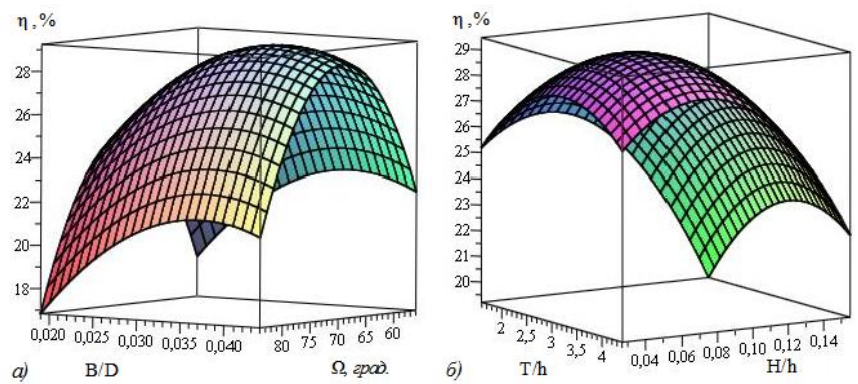

Fig. 6. Dependencies of the efficiency of disaggregation process on parameters: a) $B / D$ and $\Omega$, b) $T / h$ and $H / h$ 
The minimum of the function $\eta$ corresponds to the lowest value of the factor $B / D=0.019$ and the largest $\Omega=84$. The efficiency of the process of disaggregation increases with the increase in the width of the bands of the disaggregating device B to 0.032 , but even greater growth $\eta$ occurs with an increase

$\Omega$ from 56 to 69 degrees. Accordingly, the decrease in the efficiency of the disaggregation process occurs with an increase in the installation angle of the belts from 69 degrees to the end of the range, while a decrease $\eta$ with an increase in the $B / D$ factor value from 0.033 and further is less dramatic. With an angle of $\Omega 69$ degrees, disaggregation occurs most efficiently, and the angle between the aggregate velocity vector and the belts approaches 90 degrees.

In Fig. 6 b) the surface function of the efficiency of the process of disaggregation has values from $19.2 \%$ to $29.4 \%$. The value $\eta$ reaches its maximum value $T / h=3.15$ and $H / h=0.07$. The value $\eta$ begins to decrease when the $H / h$ parameters change from 0.07 to 0.027 and $T / h$ from 3.15 to 4.409.

Fig. 7 shows the dependencies of the efficiency of the disaggregation process on the parameters $T / h$ and $\Omega, H / h$ and $\Omega$.
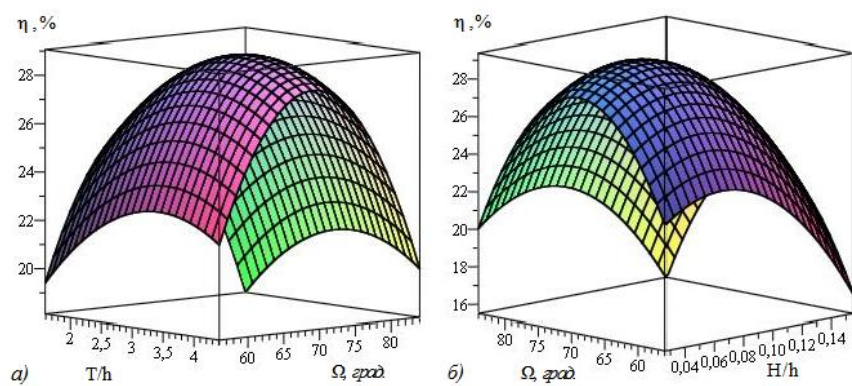

Fig. 7. The efficiency of disaggregation from the parameters a) $T / h$ and $\Omega$; b) $\Omega$ and $H / h$

The function of the efficiency of the process of disaggregation in graph 7 a) varies from 18.1 to $29.0 \%$. The greatest value $\eta$ reaches at $\Omega=68$ degrees and $T / h=3.16$. It can be seen from the graph that the change in the installation angle of belts affects the efficiency of the disaggregation process more than the parameter $T / h$. The growth of the function occurs when the angle is changed from 56 to 68 degrees and with increasing $T / h$ from the minimum value to 3.16. Then there is a sharp decrease $\eta$ with an increase $\Omega$ from 68 to 84 degrees and a gradual decrease with the growth of the factor $T / h$ to 4.409 from 3.16. The angle of installation of the belts $\Omega$ has a significant influence on the process of interaction of the device strips with the aggregates and their destruction, while the step of installing the belts affects the destruction of aggregates to a lesser degree. Fig. 7 b) represents the dependence of the change $\eta$ on factors $\Omega$ and $\mathrm{H} / \mathrm{h}$, while the efficiency varies from 15.6 to $29.3 \%$. The maximum value $\eta$ reaches at an angle of installation of belts of 72 degrees and a value of $H / h=0.096$. The minimum of the function at the maximum $H / h$ and $\Omega$. The increase in the efficiency of the disaggregation process occurs with an increase in the installation angle $\Omega$ from 56 to 71 degrees, followed a growth of $\eta$. The efficiency of the disaggregation process increases insignificantly with increasing $H / h$ from 0.027 to 0.096 , with a further increase in $H / h$, the efficiency of the disaggregation process drops sharply, a similar situation occurs with a decrease from 72 to 56 degrees. As a result of installing the device at the level of 0.096 and above, the belts are moved to the zone where aggregates are smaller, since aggregates of coarse particles have a downward trajectory, as a result of which the formation of fine particles decreases, the efficiency of the disaggregation process also decreases [11].

\section{CONCLUSION}

Separators for the separation of powdered materials are widely used to produce a product with a specified granulometric composition. Increasing the efficiency of particle disaggregation is one of the promising directions for improving the separation process. Obtained equations allow one to estimate the efficiency of particle disaggregation in a dynamic separator with a disaggregating device, and also to determine the rational angle of the installation of multi-pass belts to provide a frontal contact for maximum efficiency. Dependences of the efficiency of the process of disaggregation depending on the degree of disaggregation are obtained. Based on the results of experiments on the separation of ground made on the experimental dynamic separator with a disaggregating device, the dependence of the disaggregation efficiency on the design parameters of the device is given, which also makes it possible to determine their rational values. The practical usefulness and significance of using such device is shown.

On the basis of the mathematical description, an equation is obtained for determining the rational angle for the installation of multi-pass belts, in which a frontal contact is provided. The calculated value of the rational angle $\Omega$ for aggregates of size $315 \mu \mathrm{m}$ was 75 degree, with the aggregate size $630 \mu \mathrm{m}$ - angle of 66 degree. A mathematical expression is obtained for calculating the efficiency of the process of disaggregation by a device in the form of multi-pass belts in a dynamic separator. For aggregates of different fractions, graphical dependencies of the efficiency of disaggregation process on the degree of disaggregation are shown. Thus, the efficiency of the disaggregation process for aggregates of size $315 \mu \mathrm{m}$ with the degree of disaggregation 1.7 is $28.7 \%$.

The results of the experiment show that when a dynamic separator bench unit is equipped with a disaggregating device in the form of multi-pass belts, a large fraction of the aggregates is destroyed upon contact with its belts. Due to the destruction of coarse aggregates, the disaggregated «fine» particles are carried away by the air flow to the finished product, while the efficiency of the disaggregation process increases by $14.2 \%$ (from 14.6 to $28.8 \%$ ), which agrees with the theoretical conclusions in the methodology section.

It is established that in order to ensure maximum performance and efficiency of disaggregation at the minimum power consumption, the rational values of the design parameters of the device are:

- the angle $\Omega$ of inclination of multi-pass belts of the disaggregating device is equal to 67 ... 77 degree; 
- height of installation of the device in the separation chamber $-\mathrm{H}=\mathrm{h} \cdot(0.045 \ldots 0.091)$;

- screw pitch of the belts $-\mathrm{T}=\mathrm{h} \cdot(2.609 \ldots 3.606)$; the width of the belts is $\mathrm{B}=\mathrm{D} \cdot(0.029 \ldots 0.039)$.

\section{Acknowledgment}

The work is realized in the framework of the Program of flagship university development on the base of Belgorod State Technological University named after V.G. Shoukhov, using equipment of High Technology Center at BSTU named after V.G. Shoukhov.

\section{References}

[1] N.F. Glukharev, Dry grinding under electroneutralization conditions, $\mathrm{SPb}$.: Publishing house of Polytechnic. University, 2014.

[2] B.V. Deryagin, N.V. Churaev, V.M. Muller, Surface forces, Moscow: Nauka, 1985.

[3] M. Clark, Separation efficiency. International Cement Review (ICR), 2004.
[4] J. Haber, "Manual on catalyst characterization (Recommendations 1991)," Pure Appl. Chem, Vol. 63, pp. 1227-1246, 1991.

[5] Ivan V. Klumpar, Currier Fred N., Ring Terry A, "Air Classifiers," Chemical Engineering, pp. 77 - 92, March 31986

[6] P.A. Rebinder, Surface phenomena in disperse systems, Physicochemical mechanics. Selected works, Moscow: Nauka, 1979.

[7] N.B. Uryev, Physico-chemical dynamics of disperse systems and materials, The success of chemistry, Vol. 73: 1, pp. 39-62, 2004.

[8] E.D. Shchuki, A.V. Pertsov, E.A. Amelina, Colloid chemistry, M : Higher education. Sch., 2004.

[9] O.S. Ivanov, M.S. Vasilishin, "To an estimation of power inputs at wet grinding of a disperse material," Applied Chemistry magazine, vol. 84, issue 4, pp. 591-594, 2011.

[10] V.N. Trofimchenko, V.P. Voronov, O.S. Mordovskaya, S.I. Khanin, "Determination of the angle of installation of the beltss of the disaggregating device and its efficiency for marl separation," Scientific and theoretical magazine. Bulletin of BSTU. V.G. Shukhov, Vol. 11, pp. 114-117, 2016.

[11] V.N. Trofimchenko, O.S. Mordovskaya, S.I. Khanin, "Research of aggregates of coarse-grained marl particles and the process of their disaggregation in a separator with a device in the form of multi-pass belts," Scientific and theoretical magazine. Bulletin of BSTU. V.G. Shukhov, Vol. 12, pp. 114-120, 2016. 